\title{
Investigadores y publicaciones científicas en la UCR
}

\author{
Researchers and scientific publications at UCR \\ DOI 10.5377pc.v0i16.8092
}

Jorge Polanco Cortés ${ }^{1}$

\section{$\mathrm{PCl}$ ¿Cómo ha trabajado la UCR para fortalecer sus publicaciones científicas?}

JPC/ La Universidad de Costa Rica ha implementado varias acciones para fortalecer sus publicaciones periódicas, primeramente desde $2001^{2}$ es miembro activo de Latindex, y esto permitió establecer años después la evaluación obligatoria anual de las revistas de la UCR con los criterios Latindex. Las evaluaciones generaron la necesidad de capacitación y apoyo a las revistas, un pilar de Latindex. Gracias a este proceso anual de evaluación, las personas responsables de revistas de la Universidad entienden los procesos de evaluación de revistas y fortalecen sus revistas en una actitud de mejora continua. El cumplir los criterios Latindex permitió a algunas revistas alcanzar otras indexaciones más rigurosas.

A partir del 2012, se creó "UCR índex", un sistema de evaluación para las revistas de la Universidad de Costa Rica, ya que para este momento los criterios Latindex eran insuficientes para discriminar las revistas según calidad, considerando que la mayoría de revistas cumplían más del $90 \%$ de los criterios Latindex. UCRíndex busca crear criterios de calidad más rigurosos para las revistas UCR, que estén alineados a las tendencias mundiales de publicación y que ayuden a la revistas a prepararse para la evaluación en sistemas internacionales que comúnmente tienden a ser más rigurosos.

Este índice ha ido evolucionando a través de los años y en el 2017 lanzamos la última versión del índice con exigencias superiores a las versiones originales del UCRíndex. ${ }^{3}$

\footnotetext{
1 Jorge Polanco Cortés: Coordinador del Portal de Revistas de la Universidad de Costa Rica. Capacitador del software Open Journal System. Parte del equipo del repositorio Institucional Kérwá de la Universidad de Costa Rica, así como Editor para Costa Rica el repositorio mundial de Bibliotecología EᄀLIS.: jorge.polanco@ucr.ac.cr

${ }^{2}$ https://ucrindex.ucr.ac.cr/?p=1818

${ }^{3}$ Ver: https://ucrindex.ucr.ac.cr/?page_id $=4$
} 
En el 2013, con apoyo de Rectoría, se crearon 9 tiempos completos administrativos destinados exclusivamente para la contratación de editores administrativos (técnicos) para fortalecer las publicaciones de la Universidad y cumplir con las evaluaciones tanto de Latindex como UCR índex. Estos tiempos fueron divididos entre las revistas que mejor rendimiento han tenido en las evaluaciones. Además se destinó un tiempo completo a la coordinación del portal de revistas, que se encarga de la capacitación y acompañamiento de revistas. El establecer tiempos editoriales a las revistas buscó fortalecerlas y permitirles tramitar los artículos con revisiones por pares, concursar en índices rigurosos y cumplir con los tiempos de publicación.

Desde 2013 también se instauraron capacitaciones anuales para el fortalecimiento de las revistas, por lo que todos los años se brindan las siguientes capacitaciones:

1. Taller para nuevos editores

2. Uso del sistema Open Journal System (OJS)

3. Jornadas para personas editoras de revistas

4. Cumplimiento de criterios Latindex

$Y$ dependiendo de las necesidades, se dan capacitaciones extraordinarias orientadas o se gestionan capacitaciones según las necesidades o tendencias en la publicación de revistas, por ejemplo etiquetado XML-Jats, Correcta citación en APA, sistema de evaluación para la indexación en SCOPUS, entre otros.

Más recientemente se han creado incentivos a las personas que trabajan 0 colaboran con la revistas, por medio de nuestro sistema de incentivos. El sistema de incentivos en la UCR, llamado Régimen académico, permite a una persona docente-investigadora calificar a catedrático, por medio de sus publicaciones, evaluaciones docentes, participación académica, entre otros requisitos. La Vicerrectoría instauró un rubro que da puntaje a las personas que asumen la dirección o edición de una revistas, así como los que participan como evaluadores pares de revistas, motivando la participación en las publicaciones tanto institucionales como internacionales.

\section{PCl ¿Qué importancia tiene el UCR Índex en este proceso de fortalecimiento?}

JPC/ Muchísima importancia, puesto que nuestro sistema busca que las revistas mejoren indicadores de sistemas internacionales. EI UCR índex estimula a los 
editores a entender mejor las evaluaciones, los criterios de calidad, y a mejorar sus propias políticas, entendiendo mejor temas como propiedad intelectual, licenciamiento, preservación digital, ética en la publicación, entre otros.

\section{$\mathrm{PCl}$ ¿Cómo han evolucionado las políticas institucionales para estimular las estrategias de divulgación y publicación?}

JPC/ Creo que lo contesté en las preguntas anteriores, pero puedo aportar que hasta el 2006 no existían datos sobre la calidad de las revistas, sobre las mejoras 0 posibles impactos de la publicación de las revistas en la institución. Una vez que la Vicerrectoría de Investigación inicia con las políticas de mejora, evaluación y capacitación de las revistas institucionales empezamos a entender mejor las publicaciones, sus puntos fuertes y débiles y con eso crear estrategias para la capacitación de las personas responsables de revistas. Las evaluaciones UCR índex han cambiado al menos 5 veces desde el 2012, y esto ha significado no solo mejoras en la metodología de evaluación sino que la incorporación de criterios más rigurosos exigiendo a las revistas criterios de calidad de índices y bases de datos internacionales.

La Vicerrectoría de Investigación desde hace unos años está apuntando a una política de internacionalización de la ciencia, en el cual, se busca concursar por fondos de investigación internacionales, exportar nuestros resultados de investigación, crear productos de innovación con impacto internacional para posicionar mejor nuestra institución en el mapa internacional, esto sin dejar de lado las necesidades de la sociedad Costarricense ni la investigación regional, vital para el desarrollo de nuestros pueblos y que nos permiten comprender mejor nuestro entorno.

La Vicerrectoría también ha realizado esfuerzos para fortalecer el papel del repositorio institucional involucrándolo dentro de las gestiones institucionales. Hoy en día, la cantidad de requisitos para un trámite en Vicerrectoría de Investigación se reducen si las personas investigadoras y las unidades de investigación depositan previamente en el repositorio. También se ha impulsado la creación de fondos de estímulo para grupos de interés, potencializando la mentoría en investigación y los proyectos de investigación de excelencia.

La UCR cuenta con un equipo humano dedicado al trabajo de evaluación y consolidación de las revistas científicas institucionales que también brinda capacitaciones fuera de la institución, incluso a nivel internacional. ¿Cómo los ha 
enriquecido esta experiencia?

La Vicerrectoría de Investigación dispone de personal que está dedicado todo su tiempo a atender temas de comunicación de la ciencia, esto involucra a las personas investigadoras, para los cuales se dedican capacitaciones y acompañamiento durante el proceso de publicación, divulgación y difusión, a las personas editoras, como se mencionó anteriormente y a los gestores de sistemas, como coordinadores de repositorios, sistemas de archivo, biblioteca entre otros. La existencia de este equipo beneficia a toda la institución ya que está atenta a promover las buenas prácticas de publicación y a recomendar las mejores formas de posicionar la investigación a nivel internacional. Con esto quiero decir que no solo las revistas se benefician sino que toda la divulgación y difusión institucional.

El equipo brinda capacitación para la correcta interconexión, indexación y metaetiquetado de plataformas, lo que permite a los sistemas integrar otros con un alcance mayor. Esto permite, por ejemplo, que los documentos que integran el portal de revistas estén a su vez en el repositorio institucional de manera automática, y a su vez en la referencia, repositorio regional, y a su vez en repositorios europeos. También logramos detener la publicación de personas investigadoras de la institución en revistas depredadoras y mejorar las prácticas de inclusión de información en la web, por ejemplo los perfiles de Google Scholar de nuestras personas académicas, entre otros.

Que el equipo participe en eventos internacionales, permite actualizar las prácticas propias, validar posiciones y crear estrategias para alcanzar nuevos desarrollos. El trabajo en difusión de la ciencia no para, cada día hay nuevas formas de presentar las investigaciones y es necesario estar al día.

\section{$\mathrm{PCl}$ ¿Qué retos encuentran para mejorar la proyección de las revistas científi- cas de la UCR internacionalmente?}

JPC/ Nos hace falta tiempo para hacer mayor divulgación de la publicación, por ejemplo, es necesario más periodismo de datos, creación de blogs y wikis científicos. El incorporar la investigación institucional en espacios sociales promueve su lectura y la internacionalización de la ciencia regional.

Hay retos que como equipo no podemos resolver, sino que son las propias revistas que deben resolver como temas de calidad, políticas internas de la publica- 
ción, evaluación entre otros. Nosotros brindamos las herramientas pero si las personas responsables de la publicación no muestran interés, las revistas pueden llegar a estancarse y no crecer como se espera. Por eso, no es solo iniciar con capacitaciones o evaluaciones sino que esto debe estar inmerso en una estrategia, que esté acompañado de políticas (apoyo institucional). No sabemos si la cantidad de revistas en la institución es mucho o poco (50 revistas) pero si se puede observar que hay campos en los que al existir tantas revistas del mismo tema, pueden estarse debilitando entre sí, ya que los investigadores se dividen, los fondos se dividen y los esfuerzos se dividen.

Finalmente, otro tema que está fuera de nuestro alcance es la forma en que la ciencia se evalúa a nivel institucional o regional, y es muy importante la manera en que se realizan estas evaluaciones ya que directamente afectan las dinámicas de publicación de las personas investigadoras.

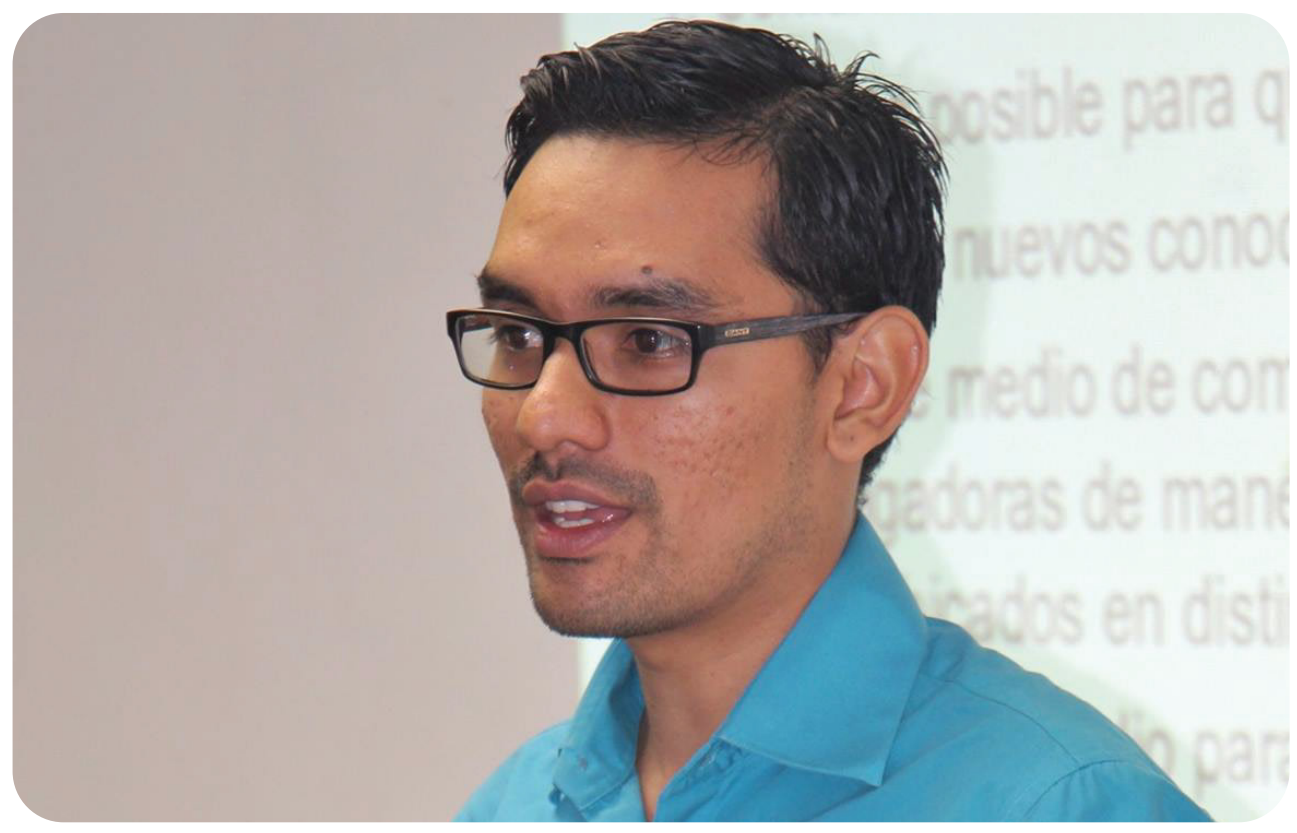

Jorge Polanco Cortés 